\title{
Prevención de la endocarditis infecciosa en niños y adolescentes con cardiopatías congénitas. Revisión de la literatura.
}

María Gabriela Acosta de Carmargo ${ }^{1}$;

Claudia Giunta Crescente';

Yamir Gamarra Arévalo3;

\section{Resumen}

Las cardiopatías congénitas incluyen anomalias en la estructura cardiaca que ocurren antes del nacimiento. Dichos defectos se dan en el feto mientras se desarrolla durante el embarazo. Este grupo de pacientes son de riesgo para contraer endocarditis infecciosa durante la consulta odontológica. Objetivo: recopilar información actualizada sobre el control odontológico para prevenir la endocarditis infecciosa en niños y adolescentes que presenten cardiopatías congénitas. Materiales y
Métodos: se realizó una búsqueda, mediante consulta electrónica en las principales bases de datos científicas Pubmed, Lilacs, Scielo, Medline, EBSCO desde 1990 a 2017 en inglés, español y portugués. Conclusión: De acuerdo a las últimas pautas mundiales cada paciente debe ser tratado de acuerdo al riesgo real de desarrollar o no EI y no debe impartirse la profilaxis antibiótica como se hacía en el pasado.

Palabras Claves: Endocarditis Infecciosa, cardiopatía congénita, niños.

Artigo de Revisão

\section{Prevenção de endocardite infecciosa em crianças e adolescentes com cardiopatias congenitas. Revisão da literatura}

\section{Resumo}

Doenças cardíacas congênitas incluem anormalidades na estrutura coronária durante a embriologia. Esses defeitos ocorrem no feto à medida que ele se desenvolve durante a gra- videz. Este grupo de pacientes corre o risco de endocardite infecciosa durante o tratamento odontológico. O objetivo deste trabalho é relembrar e atualizar as informações sobre o cuidado no atendimento odontológico para prevenção de endocardite infecciosa em crian-

\footnotetext{
1. Doctora en Ciencias Odontológicas, Especialista en Odontopediatría, Univesridad de Carabobo, Valencia, Venezuela.

2. Especialista en Odontopediatría, Valencia, Venezuela.

3. Docentes del Departamento del Niño y del Adolescente. Facultad de Odontologia. Universidad de Carabobo. Valencia, Venezuela.
} 
ças e adolescentes com cardiopatia congênita. Materiais e método: uma revisão da literatura foi realizada por pesquisa eletrônica nas bases de dados: Pubmed, Liliacs, Scielo, Medline, EBSCO de 1990 a 2017 em inglês, espanhol e português. Conclusão: De acordo com as últimas diretrizes globais, cada paciente deve ser tratado de acordo com o risco real de desenvolver, ou não, IE e a profilaxia antibiótica não deve ser administrada como foi feita no passado.

Palavras-chave: Endocardite infecciosa, doença cardíaca congênita, crianças.

\section{Prevention of infective endocarditis in children and teenagers with congenital heart disease. Literature review.}

\begin{abstract}
Congenital heart diseases include abnormalities in the cardiac structure that occur before birth. These defects occur in the fetus as it develops during pregnancy. This group of patients is at risk for infectious endocarditis during the dental visit. The aim was to recollect actual information about the dental control for prevention of infective endocarditis in children and adolescents with congenital heart disease. Materials and method:
\end{abstract}

\section{Introducción}

Las cardiopatías congénitas incluyen anomalías en la estructura cardíaca que ocurren antes del nacimiento. Dichos defectos se dan en el feto mientras se desarrolla en el embarazo. Alrededor de 500.000 adultos tienen cardiopatías congénitas en Estados Unidos. Uno de cada 100 niños tiene un defecto cardíaco debido a anomalías genéticas o cromosómicas, como el síndrome de Down. El consumo excesivo de alcohol durante el embarazo y uso de medicamentos, infecciones virales maternas como virus de rubeola o parotiditis en el primer trimestre
A literature review was done by electronic research with the data-base: Pubmed, Liliacs, Scielo, Medline, EBSCO from 1990 to 2017 in english, spanish and portuguesse. Conclusion: According to the latest global guidelines, each patient must be treated according to the real risk of developing IE or not and antibiotic prophylaxis should not be given as it was done in the past.

Key words: Infective endocarditis, congenital heart disease, children.

de embarazo, son factores de riesgo así como también se incrementa el riesgo si un padre o hermano tiene una cardiopatía congénita. ${ }^{1}$

Es conocido que un paciente comprometido sistémicamente puede presentar riesgos en su vida si presenta complicaciones a nivel bucal, como focos infecciosos o inadecuado manejo odontológico. ${ }^{2}$

El paciente con cardiopatía congénita tiene un riesgo incrementado de endocarditis infecciosa (EI) al ser atendido en el consultorio dental debido a que al existir una bacteremia, las bacterias pueden via- 
jar y adherirse en las estructuras cardíacas. Sin embargo, son variados los cambios que se han dado en los últimos años en cuanto al manejo odontológico dependiendo del riesgo del paciente, el cual determina la conducta a seguir. A pesar de que la EI no es común en la infancia, su asociación con cifras epidemiológicas en pacientes con cardiopatías congénitas no está muy clara.

Las lesiones endoteliales del corazón o de los vasos ocasionan trombos locales que pueden infectarse a partir de bacterias que pasan al torrente sanguíneo (endocarditis, flebitis); esto lo hacen especialmente los gérmenes con gran adherencia por los endotelios, como ciertos Streptococos. ${ }^{3}$ También el Staphylococcus aureus se ha convertido en una causa dominante de EI y es un predictor independiente de mortalidad. ${ }^{4}$

La EI es una enfermedad poco frecuente que, sin embargo, en el siglo XXI mantiene una elevada morbilidad (tasas de cirugía cardíaca del 50\%) y mortalidad (aproximadamente el 20\%), a pesar de los grandes progresos médicos y quirúrgicos en su diagnóstico y tratamiento. ${ }^{3}$

Muchos procedimientos dentales causan bacteremia y se creía que la misma podía llevar a EI en algunas personas. Las guías clínicas en muchos países han recomendado actualmente que antes de procedimientos dentales invasivos se administren antibióticos en personas de alto riesgo a contraer EI. ${ }^{5}$

\section{Materiales y Métodos}

Se realizó una búsqueda electrónica de artículos en revistas especializadas en Odontología y Cardiología pediátrica en las bases de datos:
Pubmed, Liliacs, Scielo, Medline, EBSCO, desde 1990 a 2017 en inglés, español y portugués. Se emplearon los siguientes términos de búsqueda: Cardiopatía congénita, Endocarditis infecciosa, manejo odontológico y paciente pediátrico. Se localizaron 205 artículos, de los cuales se tomaron en cuenta las guías practicas clínicas, estudios de cohorte y casos y controles. Se seleccionaron todos aquellos artículos que incluyeran las palabras claves mencionadas. Se excluyeron artículos que no tuvieran disponible el resumen. También se excluyeron reportes de casos clínicos.

El objetivo de esta revisión fue recopilar información actualizada sobre el control odontológico para prevenir la endocarditis infecciosa en niños y adolescentes que presenten cardiopatías congénitas.

Al tratar un paciente comprometido sistémicamente, el equipo de trabajo que lo atiende debe estar capacitado para conocer la incidencia, prevalencia, características clínicas de la enfermedad, manejo médico; así como en el área de odontología, el manejo odontopediátrico. Es necesario conocer cuáles son las patologías cardíacas que se observan más comúnmente antes de abordar al paciente, así como entender las posibles complicaciones que pudieran presentarse de no seguir las pautas ya establecidas. ${ }^{3}$

\section{Cardiopatías Congénitas (CC) más frecuentes:}

Los pacientes con CC tienen 6.5 veces más riesgo de tener una cromosomopatía asociada. La mortalidad en niños menores de 1 año supone algo más de $1 / 3$ de las muertes por anomalías congénitas y alrededor de $1 / 10$ de todas las muertes en ese periodo de la vida con una tendencia a dis- 
minuir con el tiempo debido a los avances y mejores técnicas de manejo médico y quirúrgico. La aorta bivalva es la CC más común, sin embargo, su repercusión solo se evidencia en la adolescencia o en la adultez, por lo cual la comunicación interventricular (CIV) se presenta como la más frecuente con repercusión en la edad temprana hasta en un $60 \%$, seguida por la comunicación interauricular (CIA), estenosis pulmonar, ductus, coartación de aorta, defectos del septo atrioventricular, tetralogía de Fallot, estenosis aórtica y transposición de grandes vasos. ${ }^{6}$

Adicionalmente las CC han sido clasificadas en cianóticas, con poca o ninguna cianosis y también se han clasificado según el defecto anatómico del corazón.-9

\section{Endocarditis infecciosa (EI)}

La endocarditis infecciosa es una severa infección que surge en el revestimiento del corazón con una tasa de mortalidad alta. ${ }^{5}$

La epidemiología de la EI depende del número de factores relacionados al huésped cuya prevalencia varía globalmente. La población usualmente afectada de EI son las personas mayores y enfermas, a menudo con condiciones comórbidas asociadas. El riesgo ha crecido dentro de la población joven por el uso de drogas por vía intravenosa (IV). ${ }^{10}$

En la detección de la EI, son variados los factores que influyen en el espectro clínico observado. Incluye el descenso en la incidencia de carditis reumática, aumento del uso de dispositivos intracardíacos, población con pacientes de alto riesgo (en hemodiálisis, que usan drogas IV y diabetes); así como el mejoramiento en las herramientas diagnósticas de la EI. ${ }^{11}$

\section{Profilaxis para endocarditis infecciosa: Control Odontológico}

Cuando se lesionan y sangran los endotelios o epitelios colonizados por bacterias saprófitas, estas pueden pasar al torrente sanguíneo dando lugar a pequeñas bacteremias, que podrían infectar con mayor facilidad un endocardio defectuoso que uno sano. ${ }^{12}$

En casos sintomáticos de sospecha de alguna cardiopatía, el odontólogo debe remitir al paciente al cardiólogo, realizando primero una historia médica sobre la salud general y bucal. ${ }^{13}$

Si el odontólogo ya identificó la presencia de una cardiopatía congénita, debe evaluar el estado actual del paciente (si ha sido operado o no), se realiza interconsulta con cardiólogo. ${ }^{13}$

La profilaxis antibiótica para la realización de procedimientos odontológicos del paciente con cardiopatía congénita es de la siguiente manera: se administra una dosis única de antibiótico de 30 a 60 minutos antes de atender odontológicamente al paciente. Si al odontólogo se le olvidó medicar al paciente, puede darle la dosis única de antibiótico dos horas después de haber culminado el procedimiento odontológico. ${ }^{14,15}$

Los riesgos de endocarditis infecciosa para cada paciente son diferentes según la condición sistémica o alteración que se presente. Por tal razón, se clasifican en grupos de alto riesgo, moderado riesgo y bajo riesgo (Tabla 1). Asimismo no todos los tratamientos odontológicos son iguales ni suponen el mismo riesgo de bacteremia. Y la profilaxis antibiótica también debe planificarse según las características de cada paciente, tomando en cuenta alergias e intolerancias (Tabla 2). 


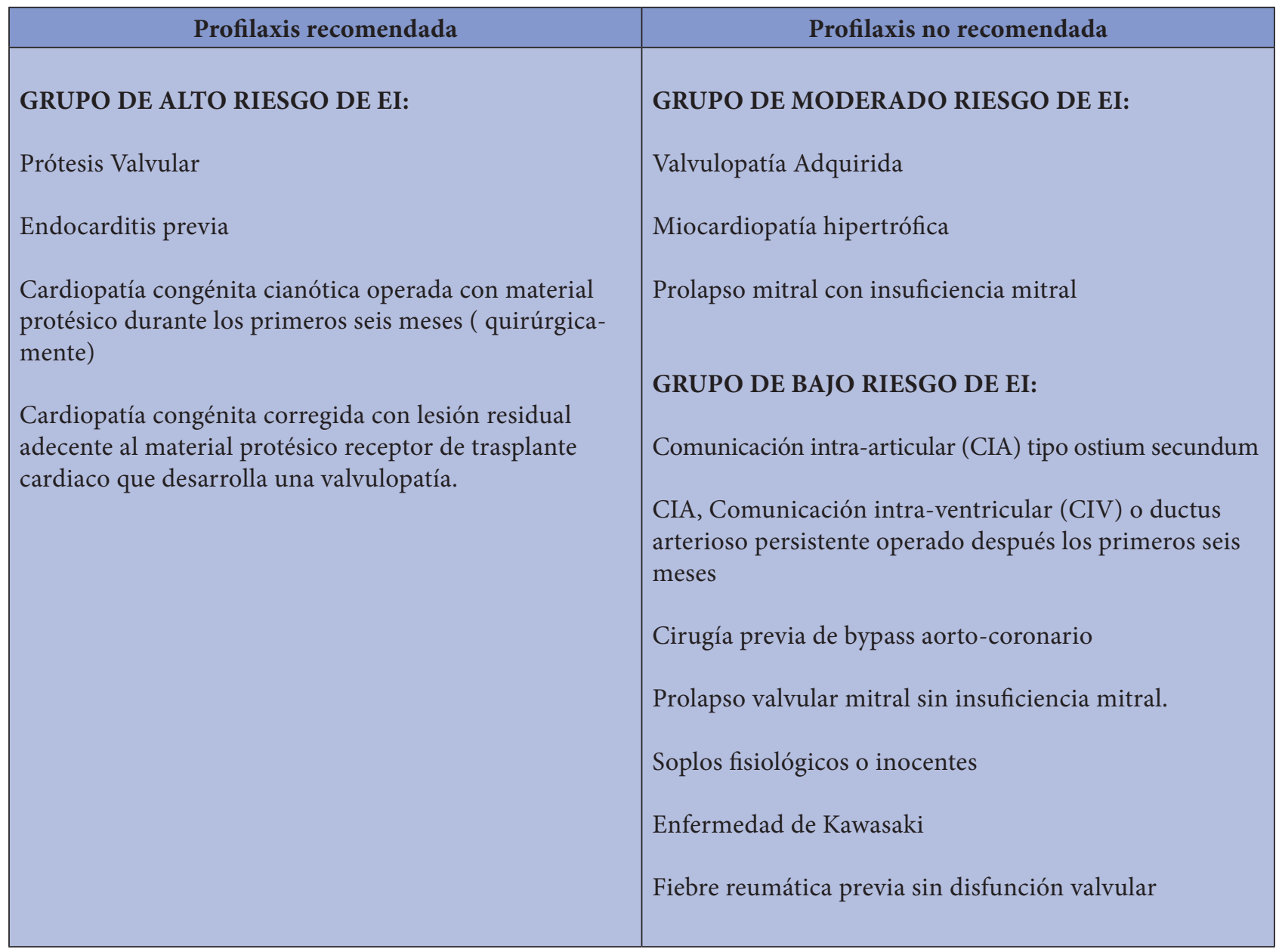

Tabla 1. Recomendaciones de profilaxis según grupo de riesgo. ${ }^{15}$

Los principales cambios en las recomendaciones por la Guía Práctica de la AHA (American Heart Association) actualizadas en el 2008 incluyen lo siguiente: (1) El comité concluyó que solo una cantidad extremadamente pequeña de casos de EI podría prevenirse mediante profilaxis antibiótica para procedimientos dentales, incluso si dicha terapia profiláctica fuera 100 por ciento efectiva. (2) La profilaxis de IE para procedimientos dentales debe recomendarse solo para pacientes con afecciones cardíacas subyacentes asociadas con el mayor riesgo de resultados adversos de la EI. (3) Para los pacientes con estas afecciones cardíacas subyacentes, se recomienda la profilaxis para todos los procedimientos dentales que implique la manipula- ción del tejido gingival o la región periapical de los dientes o la perforación de la mucosa oral. (4) No se recomienda la profilaxis basada únicamente en un mayor riesgo de por vida de adquirir IE. (5) No se recomienda la administración de antibióticos únicamente para prevenir la endocarditis en pacientes sometidos a un procedimiento genitourinario o del tracto gastrointestinal. Estos cambios pretenden definir con mayor claridad cuándo se recomienda o no la profilaxis de IE y proporcionar recomendaciones globales más uniformes y coherentes. ${ }^{16}$

El Comité señala importante identificar estas condiciones cardíacas como riesgo de desarrollar EI: Enfermedad cardíaca valvular adquirida con es- 


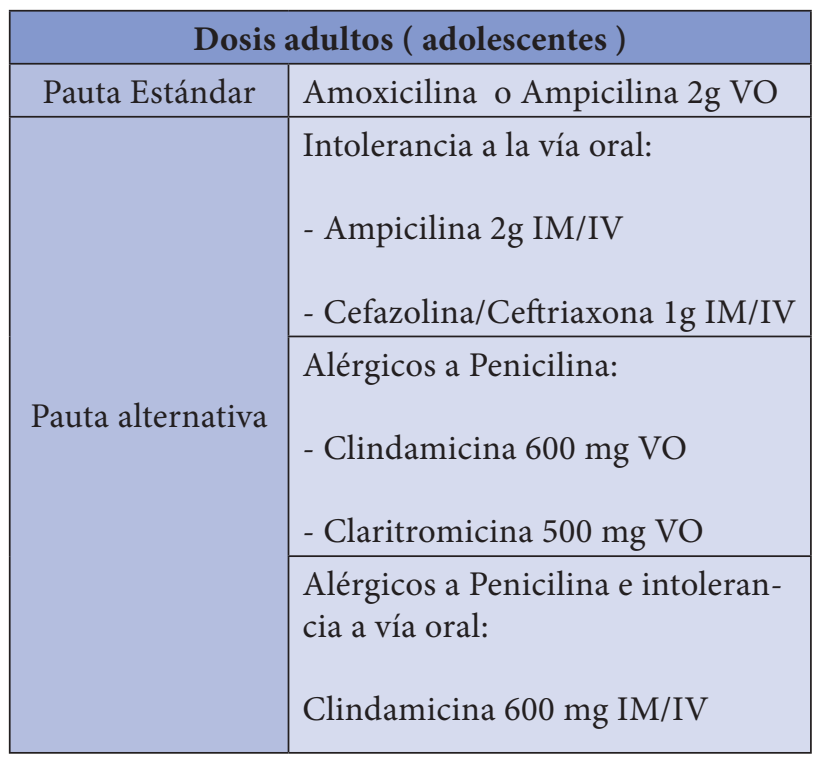

\begin{tabular}{|l|l|}
\hline \multicolumn{2}{|l|}{ Dosis pediátrica } \\
\hline Pauta Estándar & $\begin{array}{l}\text { Amoxicilina o Ampicilina } 50 \mathrm{mg} / \mathrm{kg} \\
\text { VO }\end{array}$ \\
\hline \multirow{3}{*}{ Pauta alternativa } & $\begin{array}{l}\text { Intolerancia a la vía oral: } \\
\text { - Ampicilina } 50 \mathrm{mg} / \mathrm{kg} \mathrm{IM} / \mathrm{IV}\end{array}$ \\
\cline { 2 - 3 } & $\begin{array}{l}\text { Alérgicos a Penicilina: } \\
\text { - Claritromicina } 15 \mathrm{mg} / \mathrm{kg}\end{array}$ \\
\cline { 2 - 2 } & $\begin{array}{l}\text { Alérgicos a Penicilina e intolerancia } \\
\text { a vía oral: } \\
\text { Clindamicina } 20 \mathrm{mg} / \mathrm{kg} \mathrm{IM} / \mathrm{IV}\end{array}$ \\
\hline
\end{tabular}

Tabla 2. Dosis de antibióticos para profilaxis de la endocarditis infecciosa. ${ }^{15}$

tenosis o regurgitación, reemplazo valvular, cardiopatías congénitas estructurales incluidas las afecciones estructurales corregidas quirúrgicamente o paliadas, pero excluyendo la comunicación interauricular aislada, el defecto septal ventricular completamente reparado o el ductus arterioso permeable completamente reparado, y los dispositivos de cierre que se consideran endotelializados, CC estructural con EI previa y cardiomiopatía hipertrófica. ${ }^{17-18}$

Anteriormente se daban recomendaciones acerca de cuáles procedimientos odontológicos requerían profilaxis antibiótica (exodoncias, colocación de bandas, anestesia intraligamentosa, endodoncias, tartrectomias) y cuáles no (toma de impresiones, aplicación de flúor, toma de radiografías, operatoria dental sin riesgo a exposición pulpar, anestesia local, sellantes sin aislamiento $)^{15}$. Actualmente la recomendación para la profilaxis comprende si hay involucramiento de tejido gingival, periapical o perforación de la mucosa bucal. ${ }^{16}$
Los pacientes que se someten a un procedimiento del tracto respiratorio que involucra incisión o biopsia de la mucosa respiratoria también requerirán esta nueva recomendación de la AHA. ${ }^{19}$

Consejos que deben ser impartidos a los pacientes por el personal odontológico.

$\mathrm{Al}$ momento de atender un paciente con riesgo de EI es importante ofrecer una información clara y consistente acerca de la prevención de la EI, recalcando los beneficios y riesgos de la profilaxis antibiótica e incluyendo una explicación de por qué la profilaxis antibiótica no es más recomendada de forma rutinaria. Las razones son: debido a que la efectividad clínica no está comprobada, no es costo-efectiva, y comparada con ninguna profilaxis, la profilaxis antibiótica ha llevado a más muertes por anafilaxis.

Es importante enfatizar la importancia de mantener una buena salud bucal. 
Señalar los síntomas que pueden indicar la presencia de EI y cuando buscar ayuda de un experto.

Explicar el riesgo potencial de procesos invasivos no médicos como piercing o tatuajes. 17-18

\section{Profilaxis en pacientes con riesgo de EI}

La profilaxis antibiótica contra la EI no está recomendada en las siguientes circunstancias:

- Pacientes bajo procedimientos dentales.

- Personas con procedimientos no dentales en los siguientes sitios: tracto gastrointestinal bajo y alto, tracto genitourinario (incluyendo procedimientos urológicos, ginecológicos y obstétricos y parto), tracto respiratorio alto y bajo (que incluye procedimientos en oído, nariz y garganta y broncoscopia)

Los enjuagues con clorehexidina no deben ser ofrecidos como profilaxis contra EI a personas con riesgo de EI que reciben procedimientos dentales.

Investigar de forma rápida y apropiada si existe algún episodio de infección para reducir el riesgo subsecuente de desarrollar EI.

Si una persona con riesgo de EI está recibiendo terapia antimicrobiana porque tiene un procedimiento gastrointestinal o genitourinario en un sitio donde se sospecha de infección, la persona deberá recibir un antibiótico que cubra los organismos que causan EI. ${ }^{17-18}$

\section{Discusión}

Impartir conocimiento sintetizado es esencial para avanzar en la práctica clínica de las ciencias médicas a través de investigación con evidencia científica, esto ayudará de manera eficiente en la parte clínica durante la toma de decisiones basada en evidencias. ${ }^{20-21}$ Lamentablemente al analizar muchos de los estudios clínicos realizados, los mismos carecen de diseños apropiados que garanticen su eficacia.

La evidencia para el uso de profilaxis antibiótica es limitada, heterogénea y la calidad metodológica de muchos estudios es pobre. La bacteremia post-procedimiento no es un buen punto determinante para endocarditis infecciosa. Dados los desafíos logísticos de ensayos aleatorios o estudios de casos y controles de alta calidad, estos ayudarían notablemente a evaluar el papel de los procedimientos odontológicos en la causa de la endocarditis infecciosa y la eficacia comprobada de la profilaxis antibiótica en su prevención. ${ }^{22}$

Recientemente se concluyó que la IE es mucho más probable que resulte de la exposición frecuente a bacteriemias aleatorias asociadas con actividades diarias que de las bacteriemias causadas por procedimientos médicos invasivos. Una buena higiene oral y la erradicación de la enfermedad dental es la herramienta más importante para prevenir. ${ }^{23}$

La reciente guía práctica internacional recomienda un uso mucho más limitado de profilaxis antibiótica contra la $\mathrm{EI}^{18}$ con lo que se espera una reducción en la resistencia a los antibióticos como resultado de la profilaxis antibiótica previa para la profilaxis IE realizada en los últimos años. ${ }^{19}$ 
Utilizando las nuevas pautas de las prácticas clínicas, ya han sido reportados cambios en los resultados al disminuir la profilaxis antibiótica. DeSimone et al. consiguieron una reducción significativa en la profilaxis antibiótica en pacientes en un grupo de riesgo moderado antes de procedimientos dentarios invasivos, destacando que después de la publicación de la guía de la AHA en 2007 hubo una reducción significativa en la profilaxis antibiótica. Estos hallazgos pueden usarse para una retroalimentación y educación en odontólogos envueltos en la decisión de usar profilaxis para sus pacientes. ${ }^{24}$

Los procedimientos invasivos cardíacos y no los procedimientos dentales están más asociados significativamente a EI en niños con cardiopatías congénitas $^{25}$. Aunque también cada caso debe ser analizado de forma individual, junto con el cardiólogo, para determinar el riesgo y beneficio de la suspensión de los anticoagulantes. ${ }^{15}$

La relación entre la salud bucal y la salud sistémica debe reforzarse constantemente, especialmente en los padres de niños con enfermedades cardíacas. Por lo tanto, los odontopediatras, cardiólogos y otros profesionales de la salud asociados deben trabajar juntos para educar a los niños afectados con cardiopatías congénitas y sus padres, mejorando la calidad de vida en esta población vulnerable. ${ }^{26}$

Los resultados de Koerd et al. mostraron una ausencia de información en padres de niños con enfermedades cardíacas en cuanto a prevención e higiene bucal. Tampoco tenían conocimiento de la profilaxis antibiótica y la medicación dada. Además las medidas preventivas no fueron dadas de acuerdo a la guía actual. Ellos concluyeron que el conocimiento acerca de la salud bucal en esta población debía mejo- rar y recomendaron coordinar sistemas educativos en etapas tempranas en centros especializados que involucraran cardiólogos pediatras y odontopediatras. ${ }^{27}$

En el manejo odontológico de la endocarditis infecciosa, la enfermedad que más debe prevenirse es la caries dental. ${ }^{12} \mathrm{El}$ conocimiento y las actitudes de los padres sobre la importancia de la atención de la salud bucal y sus temores sobre el tratamiento dental influyen en la atención dental de sus hijos. Por lo tanto, las actitudes de los padres y los niños hacia la salud bucal deben ser mejorados. Para lograr este objetivo, se requieren programas integrales de educación sobre salud bucal para los niños y sus padres. ${ }^{28}$ La higiene bucal diaria, la evaluación y el tratamiento regulares por parte de un odontólogo son esenciales en la prevención de la endocarditis infecciosa, ${ }^{29}$ ya que el mantenimiento de la salud bucal es más efectivo para reducir el riesgo de EI que los antibióticos profilácticos para procedimientos dentales. ${ }^{30}$

La salud bucal está íntimamente relacionada con la salud sistémica. La periodontitis, enfermedad crónica inflamatoria con alta prevalencia mundial interactúa con muchas enfermedades. Es un factor de riesgo en la compleja patogénesis de la diabetes mellitus y la enfermedad cardiovascular y juega un importante rol en el desarrollo de endocarditis y neumonía recurrente en personas mayores. ${ }^{31}$ Sin embargo, en los pacientes jóvenes no debe esperarse que la enfermedad periodontal se instale, deben mejorarse las medidas de prevención en el tejido gingival y periodontal para evitar su padecimiento.

Uno de los puntos más importantes en la actualidad en cuanto a prevención de EI, es que 
no existe evidencia acerca de si el uso de la profilaxis antibiótica es efectiva o no en personas de riesgo que van a ser sometidos a procedimientos dentales invasivos. No está claro si los riesgos potenciales y costo de administración de antibióticos compensan los efectos beneficiosos. De forma ética, cada practicante de la odontología debe discutir los potenciales riesgos y beneficios de la profilaxis antibiótica con los padres antes de tomar una decisión en cuanto a la administración. ${ }^{5}$

Es muy probable que este cambio notable en las directrices provoque un debate en la literatura médica; además, por primera vez, este cambio permite realizar estudios doble ciego aleatorizados, controlados con placebo para evaluar la eficacia de la profilaxis antibiótica de la EI. ${ }^{23}$

\section{Conclusiones}

Es importante tomar medidas preventivas para mejorar la salud bucal y que los padres tomen conciencia y conocimiento acerca de lo crucial que puede ser esta en la vida del paciente pediátrico. El odontopediatra no debe perder la oportunidad de educar a los padres de niños con CC en cuanto a higiene bucal y prevención de enfermedades infecciosas en la cavidad bucal, fomentando así un tratamiento de tipo integral, minimizando y previniendo complicaciones futuras como lo es la endocarditis infecciosa en pacientes con riesgo. De acuerdo a las últimas pautas mundiales cada paciente debe ser tratado de acuerdo al riesgo real de desarrollar o no EI y no debe impartirse la profilaxis antibiótica como se hacia en el pasado.

\section{Referencias bibliográficas}

1- Sun R, Liu M, Lu L, Zheng Y, Zhang P. Congenital heart disease: causes, diagnosis, symptoms, and treatments. Cell Biochem Biophys. 2015; 72: 857-60.

2- Nicolosi L. Atención odontológica integral a pacientes con cardiopatías congénitas del adulto. Rev Hosp Niños 2017; 59: 259-65.

3- Acosta MG, Bolívar M, Giunta C, Mora K. Manejo odontológico de pacientes pediátricos comprometidos sistémicamente. Revisión bibliográfica. Rev Odontoped Latinoam 2015; 5: 5-8

4- Cabell CH, Jollis JG, Peterson GE, Corey GR, Anderson DJ, Sexton DJ, Woods CW, Reller LB, Ryan T, Fowler VG Jr. Changing patient characteristics and the effect on mortality in endocarditis. Arch Intern Med. 2002;162: 90-4.

5- Glenny AM, Oliver R, Roberts GJ, Hooper L, Worthington HV. Antibiotics for the prophylaxis of bacterial endocarditis in dentistry. Cochrane Database Syst Rev. 2013;(10):CD003813

6- Madrid A, Restrepo JP. Cardiopatias congenitas. Revista Gastrohnup 2013 (15) Suple: S56-S7

7- Magliola R, Althab M, Charroqio A, Moreno G, Balestrini M, Landry L, Vassallo JC, Salgado G, Suarez J, Laura JP. Cardiopatía Congénita: actualización de resultados quirúrgicos en un Hospital Pediatrico 1994-2001. Arch Argent Pediatr 2004; 102: 110-4.

8- Guerchicoff M, Marantz P, Infante J, Villa A, Gutierrez A, Montero G. Evaluación del impacto del diagnóstico precoz de las cardiopatías congénitas. Arch Argent Pediatr 2004; 102: 445-50.

9- Falces C, Miro J. Prevención de la endocarditis Infecciosa: entre el avance en los conocimientos científicos y la falta de ensayos aleatorizados. Rev Esp Cardiol 2012; 65: 1072-4 
10- Fatima S, Dao B, Jameel A, Sharma K, Strogatz D, Scribani M, Rammohan HRS. Epidemiology of Infective Endocarditis in Rural Upstate New York 2011-2016. J Clin Med Res. 2017; 9: 754-8.

11-Slipczuk L, Codolosa JN, Davila CD, Romero-Corral A, Yun J, Pressman GS, Figueredo VM. Infective endocarditis epidemiology over five decades: a systematic review. PLoS One. 2013;8: e82665

12- Herranz J. Control de los niños con cardiopatía congénita en atención primaria. Rev Pediatr Aten Primaria 2009; 11: 638-55.

13- Cortes JM, Ayala C, Cortes R, et al. Protocolo de atención a niños y adolescentes con cardiopatía congénita en odontopediatría. Revisión de la literatura. Rev Latinam Odontoped 2015; 5: 1-4.

14- Gonzales JA, Salazar L, Salazar C. Cardiopatías congénitas en el Hospital de México. Rev Med Costa Rica; 2000; $551:$ 47-56. 15- Habib G, Lancellotti P, Antunes M, Bongiorni MG, Casalta JP, del Zotti F, Dulgheru R, El Khoury G, Erba PA, Lung B, Miró JM, Mulder B, Plonska-Gosciniak E, Price S, Roos-Hesselink J, Snygg-Martin U, Thuny F, Tornos P, Vilacosta I, Zamorano JL. Guía de la Sociedad Española de Cardiología sobre el tratamiento de la endocarditis infecciosa. Rev Esp Cardiol. 2016; 69: 49-69.

16- Wilson W, Taubert KA, Gewitz M, Lockhart PB, Baddour LM, Levison M, Bolger A, Cabell CH, Takahashi M, Baltimore RS, Newburger JW, Strom BL, Tani LY, Gerber M, Bonow RO, Pallasch T, Shulman ST, Rowley AH, Burns JC, Ferrieri P, Gardner T, Goff D, Durack DR. Prevention of infective endocarditis: guidelines from the American Heart Association: a guideline from the American Heart Association Rheumatic fever, endocarditis and Kawasaki Disease Commitee, Council on Cardiovascular Disease in the young and the Council on Clinical Cardiology, Council on Cardiovascular Surgery and Anesthesia, and the Quality of Care and outcomes Research Interdisciplinary Working Group. J Am Dent Assoc. 2008; 138: 253.

17- Richey R, Wray D, Stokes T. Prophylaxis against infective endocarditis: summary of NICE guidance. BMJ 2008; 336: 770-1

18- National Institute for Health and Clinical Excellence. Prophylaxis against infective endocarditis 2008. (NICE clinical guideline No. 64) www.nice.org.uk/CG064

19- Sanchez-Rodriguez F, Rivera R, Suarez-Gonzalez J, Gonzalez-Claudio G. Prevention of infective endocarditis: a review of American Heart Association guidelines. Bol Asoc Med P R. 2008; 100: 25-8.

20- Colquhoun H, Levac D, O'Brien KK, Straus S, Tricco AC, Perrier L. Scoping reviews: Time for clarity in definition, methods, and reporting. J Clin Epidemiol. 2014; 67: 1291-4.

21- Fleming PS, Koletsi D, O'Brien K, Tsichlaki A, Pandis N. Are dental researchers asking patient-important questions?. A scoping review. J Dent. 2016; 49: 9-13.

22- Cahill TJ, Harrison JL, Jewell P, Onakpoya I, Chambers JB, Dayer M, Lockhart P, Roberts N, Shanson D, Thornill M, Heneghan CJ, Prendergast BD. Antibiotic prophilaxis for infective endocarditis: a systematic and meta-analysis. Heart. 2017; 103: 937-44.

23- Gottesman-Yekutieli T, Siegman-Igra Y. A revolutionary change in the recommendations for antimicrobial prophylaxis of infective endocarditis (ie). Harefuah. 2008; 147: 532-5, 573

24- DeSimone DC, El Rafei A, Challener DW, Carr AB, Kelly JA, Rocca WA, St Sauver JL, Bock-Goodner CM, Lahr BD, Steckelberg JM, Wilson WR, Baddour LM. Effect of American Heart Association 2007 Guidelines on the Practice of Dental Prophylaxis for the prevention of infective endocarditis in Olmsted County, Minnesota. Mayo Clin Proc. 2017 25- Sun LC, Lai CC, Wang CY, Wang YH, Wang JY, Hsu YL, Hu YL, Wu ET, Lin MT, Sy LB, Chen L. Risk factors for infective endocarditis in children with congenital heart diseases - A nationwide population-based case control study. Int J Cardiol. 2017; 248: 126-30 
26- Garrocho-Rangel A, Echavarría-García A-C, Rosales-Bérber M-Á, Flores-Velázquez J, Pozos-Guillén A. Dental management of pediatric patients affected by pulmonary atresia with ventricular septal defect: A scoping review. Medicina Oral Patología Oral y Cirugía Bucal.

27- Koerdt S, Hartz J, Hollatz S, Frohwitter G, Kesting MR, Ewert P, Oberhoffer R, Deppe H. Dental prevention and disease awareness in children with congenital heart disease. Clin Oral Investig. 2017

28- Reshma Suvarna, Kavita Rai, Amitha M Hegde. Knowledge and Oral Health Attitudes among Parents of Children with Congenital Heart Disease. Int J of Clinic Pediatr Dentist 2001; 4: 25-8.

29- De Munter P, Peetemans W, Declerk D; Groupe de travail ‘Prophylaxie de l'endocardite infectieuse-groupe de direction 'Therapie Antibiotique', Cliniques Universitires Leuven. Prevention of endocarditis: changes in the recommendations. Rev Belge Med Dent (1984). 2008; 63: 29-35.

30- Farbod F, Kanaan H, Farbod J. Infective endocarditis and antibiotic prophylaxis prior to dental/oral procedures: latest revision to the guidelines by the American Heart Association published April 2007. Int J Oral Maxillofac Surg. 2009; 38: 626-31.

31- Dorfer C, Benz A, Aida J, Campard G. The relationship of oral health with general Health and NCDs: a brief review. Int Dent J. 2017; 67 Suppl 2: 14-8.

Recibido: 02/10/2017

Aceptado: 13/11/2017

Correspondencia: María Gabriela Acosta de Carmargo. gabrieladecamargo@yahoo.com 